\title{
Bilateral Proliferative Retinopathy
}

National Cancer Institute

\section{Source}

National Cancer Institute. Bilateral Proliferative Retinopathy. NCI Thesaurus. Code C155316.

Proliferative retinopathy occurring in both eyes. 